\title{
Research on Novel Fuzzy Control Strategy of Hybrid Electric Vehicles Based on Feature Selection Genetic Algorithm
}

\author{
Tianjun Zhu, ${ }^{1 *}$ Linglong Wang, ${ }^{2}$ Xiaoxiang $\mathrm{Na}^{3}$ \\ Tunglung $\mathrm{Wu},{ }^{1}$ Wei Hu,${ }^{4}$ and Rouchun Jiang ${ }^{1}$ \\ ${ }^{1}$ Department of Mechanical and Automotive Engineering, Zhaoqing University, Zhaoqing 526061, China \\ ${ }^{2}$ China Automotive Technology and Research Center Co., Ltd., Tianjin 300300, China \\ ${ }^{3}$ Department of Engineering, University of Cambridge, Cambridge, CB2 1PZ, United Kingdom \\ ${ }^{4}$ College of Mechanical and Equipment Engineering, Hebei University of Engineering, \\ Handan 056021, China
}

(Received May 17, 2020; accepted November 16, 2020)

Keywords: fuzzy control strategy, feature selection genetic algorithm, fuel economy, emission

We propose a novel fuzzy control strategy for hybrid electric vehicles (HEVs) based on the feature selection genetic algorithm of multivariate data, which greatly shortens the selection time of the optimal parameters of the traditional genetic algorithm. Firstly, we take the fuel consumption and emission of an HEV as the optimization index, and develop a novel fuzzy control method considering parameters of the fuzzy controller with high correlation with the objective function, in which the membership function parameter is optimized by the feature selection genetic algorithm. Finally, the performances of the fuzzy control strategy for an HEV and the novel fuzzy control strategy optimized by the feature selection genetic algorithm under the New European Driving Cycle (NEDC) and Urban Dynamometer Driving Schedule (UDDS) cycle conditions are analyzed and compared. The results show that the proposed fuzzy control can greatly improve the fuel economy and reduce the emission of HEVs.

\section{Introduction}

In recent years, with the energy crisis becoming increasingly severe, hybrid electric vehicles (HEVs) with high efficiency, low energy use, and low emissions have been an important technology toward solving the energy crisis and reducing environmental pollution. Achieving the balance of emissions and driving range has become a bottleneck restricting the further development of HEVs.

Many scholars and research institutions have carried out considerable research on optimization algorithms for the emissions and driving range of HEVs. ${ }^{(1-6)}$ Among them, various intelligent algorithms are the most prominent, such as fuzzy control algorithms. Fuzzy control is rule-based control, which directly uses language-based control rules. A fuzzy control system has strong robustness, and the influence of disturbances and parameter changes on the control effect is greatly weakened. It is especially suitable for the control of nonlinear, time-varying, and time-delay systems.

*Corresponding author: e-mail: zhutianjun@zqu.edu.cn https://doi.org/10.18494/SAM.2021.3013 
However, fuzzy controllers are more mature and popular, and there are many methods of parameter optimization. ${ }^{(7-10)}$ For example, a trial and error method with repeated design and debugging can be used to find a more reasonable solution to specific problems. However, a trial and error method cannot guarantee the selection of the most appropriate parameter values, and the data extraction time is long. Yi et al. ${ }^{(1)}$ proposed a method of automatic design of fuzzy controllers, and optimized the parameters of a fuzzy controller with a genetic algorithm of multigroup coevolution. Lucio et al. ${ }^{(12)}$ used a genetic algorithm to realize the nondifferentiable and highly nonlinear optimization of fuzzy controller parameters. This algorithm searched for all the solutions in the feasible solution space without gradient information of the objective function, without falling into the trap of a local minimum value or the dead cycle phenomenon. A genetic algorithm is a global optimization algorithm, ${ }^{(13)}$ which is suitable for the parameter optimization of an HEV fuzzy controller.

However, there are some problems to be solved when the traditional genetic algorithm is used to optimize a fuzzy controller. When the traditional genetic algorithm is used to optimize the fuzzy control rules and membership function of a fuzzy controller, because there are many parameters to be optimized, including the number of fuzzy rules, the structure of the fuzzy rules, and the shape and division of the membership function, the structure of the gene string becomes more complex and the size of the initialization population is increased when coding the parameters to be optimized. ${ }^{(14)}$ The main reason for this is that many of the parameters to be optimized in a fuzzy controller are not closely related to the objective function to be calculated, and most of them have a low correlation with the objective function to be optimized. ${ }^{(15)}$ Therefore, it is suggested that an improved fuzzy control algorithm based on the genetic algorithm can be applied to the design of an HEV control algorithm to reduce the strong nonlinearity of the system, eliminate dead cycles, and effectively improve the accuracy of vehicle dynamic control.

We have developed a novel fuzzy control method considering the parameters of a fuzzy controller with high correlation with the objective function, in which the membership function parameter is optimized by the feature selection genetic algorithm. A genetic algorithm based on the feature selection of multivariate data is proposed. The proposed algorithm first evaluates the features of the dimension, adjusts the weight of each feature according to the difference between similar nearest neighbors and dissimilar nearest neighbors of multivariate data, and guides the search of the genetic algorithm on the basis of the feature weight, so as to improve the search ability of the proposed algorithm and the accuracy of the obtained features. Then, the fitness (or objective) function of features is calculated by combining the feature weights, and the fitness is used as the evaluation index to start the genetic algorithm. The optimal feature subset is obtained, and the efficient and accurate feature selection of multivariate data is finally realized. Compared with the traditional algorithm, ${ }^{(11-15)}$ the proposed algorithm greatly shortens the selection time.

In this paper, the novel fuzzy control strategy based on the feature selection genetic algorithm is applied to the design of an HEV energy management control algorithm. The structure of the paper is as follows. The genetic fuzzy optimization method based on feature selection is developed in Sect. 2. Section 3 focuses on the experimental parameter setting. Experimental results are presented in Sect. 4. Finally, the conclusions are given in Sect. 5. 


\section{Genetic Fuzzy Optimization Method Based on Feature Selection}

The genetic fuzzy control algorithm based on feature selection combines the genetic algorithm based on feature selection with the fuzzy control system, ${ }^{(16)}$ optimizes the fuzzy control strategy, and applies the optimization results to the actual control process. In this paper, the feature selection genetic algorithm is used to optimize the membership function parameter.

\subsection{Design of genetic algorithm based on feature selection}

The flow of the genetic algorithm based on feature selection is shown in Fig. 1. From Fig. 1, it can be seen that the optimization process based on the feature selection genetic algorithm of multivariate data is a typical iterative process. The basic steps are as follows:

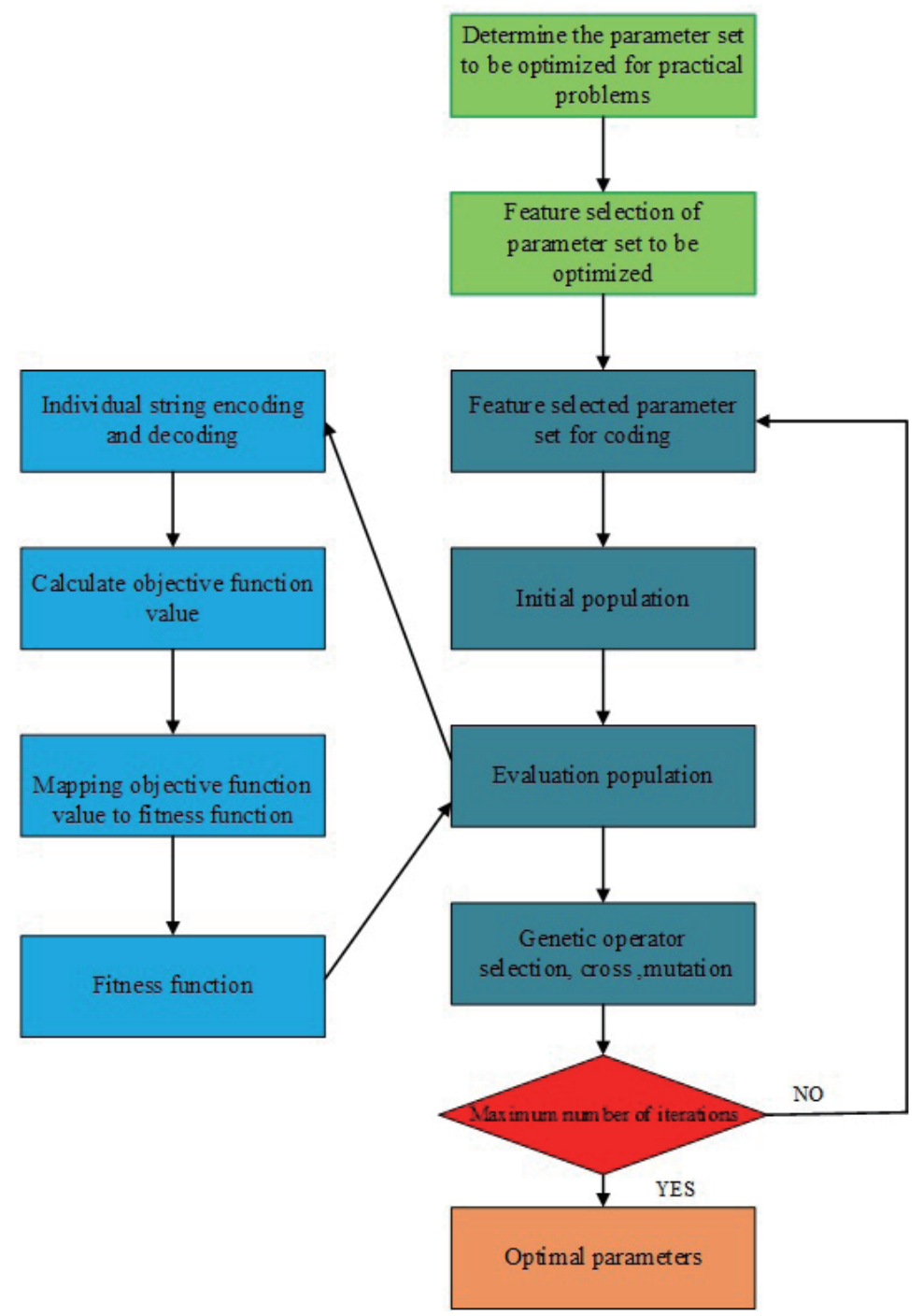

Fig. 1. (Color online) Flow chart of genetic algorithm based on feature selection of multivariate data. 
(1) The $m$ file (the text file used to save a MATLAB source program) of fuzzy controller parameters is selected by using the characteristics compiled by MATLAB software, and the parameters to be optimized are optimized.

(2) In accordance with the coding strategy, the initial population is randomly generated.

(3) The individual strings in the population are encoded and decoded, and the minimum value of the objective function, namely, the fitness function, is calculated to evaluate the population and ensure the orientation of the population.

(4) The genetic strategy and genetic operator are determined, including the population size, selection, crossover and mutation methods, and the genetic operation parameters such as crossover probability and mutation probability.

(5) It is judged whether the population performance meets the optimization index or the maximum number of iterations has been reached. If these conditions are not satisfied, the algorithm returns to step (3).

Compared with the traditional genetic algorithm, the genetic algorithm based on feature selection adds a process of feature selection optimization. In addition, the basic process of the genetic algorithm based on feature selection is essentially the same as that of the traditional genetic algorithm. The parameters of the problem to be solved are encoded to form the initial population, and the individuals in the population are selected, crossed, and changed. According to the adaptability of individuals to the environment, the process of survival of the fittest is realized. From the perspective of optimization of the search, the genetic operation can optimize the solution of the problem from generation to generation until the maximum number of iterations is reached, and the best parameters for the optimization problem can be found.

In summary, to solve the problem that the feature dimension is too high in feature-based vehicle sensor information filtering of HEVs, which affects the classification and filtering, a genetic algorithm is used for feature selection, the optimal solution is searched for by genetic operations, and the genetic operator is dynamically adjusted according to the population evolution. In order to take into account the operational efficiency and classification accuracy, feature selection is needed, which involves selecting some of the most effective data from a group of vehicle sensor data (using multivariate data analysis) with the greatest impact on the final objective function to form an optimal feature.

In this paper, the optimal parameter of feature selection, i.e., the supercapacitor power distribution factor $K_{u c}$, is selected. In the fuzzy controller, $K_{u c}$ is determined by the output membership function gaussian $\{x, c, \sigma\}$. The Gauss-type membership function in Eq. (1) needs the two parameters, $c$ and $\sigma$, to be determined together,

$$
\operatorname{gaussian}\{x, c, \sigma\}=e^{-\frac{1}{2}\left(\frac{x-c}{\sigma}\right)^{2}},
$$

where parameter $c$ determines the position of the Gaussian function and $\sigma$ affects the coverage area of the fuzzy subset. By adjusting the values of $c$ and $\sigma$, the membership function of the fuzzy controller is optimized and the final output of the fuzzy controller is determined. 
The genetic algorithm based on feature selection cannot directly deal with the parameters of the membership function, so it is necessary to transform the parameters of the membership function into chromosomes composed of gene strings in the genetic space by coding. If a string is used to represent a membership function parameter on the corresponding domain, and these strings are connected in an appropriate form, the membership function of each fuzzy subset on the domain can be represented. ${ }^{(17)}$ In order to solve the problem of coding membership function parameters, we should first find a suitable coding method for a single membership function parameter. The specific coding method is as follows.

Suppose that the range of the supercapacitor power distribution factor $K_{u c}$ to be optimized is

$$
K_{u c_{-} \min } \leq K_{u c} \leq K_{u c_{-} \max } .
$$

$K_{u c}$ is represented by $n$-bit binary number $b$, then $b$ can be determined by the following formula. In this paper, we string all binary numbers of parameters to be optimized into a binary string and $\operatorname{set} n=7$.

$$
b=\left(2^{n}-1\right) \frac{K_{u c}-K_{u c_{-} \min }}{K_{u c_{-} \max }-K_{u c_{-} \min }}
$$

The optimization effect of the genetic algorithm based on feature selection is closely related to the selection of the objective function, and the fitness function in the genetic process can be set as the objective function of the HEV. In this paper, the fuel consumption and emission values of the HEV are selected as the objective function, and the objective function is established and optimized by varying the weight ratio. The specific objective function is

$$
\begin{gathered}
\underset{X \in \Omega}{\min J(X)}=\left[\operatorname{Fuel}_{\text {eng }}(X), F C R(X), C O(X), H C(X), N O_{x}(X)\right], \\
\text { s.t. } g_{j}(X) \geq 0, j=1,2, \ldots, J
\end{gathered}
$$

where $J(X)$ is the control objective function of the HEV; $g_{j}(X) \geq 0, j=1,2, \ldots, J$ is a set of nonlinear inequality constraints including the dynamic performance indicators of HEVs. $X$ is the set of parameters to be optimized for practical problems; $\Omega$ is the feasible solution space; Fuel $_{\text {eng }}(X)$ is the fuel consumption of the internal combustion engine; $F C R(X)$ is the equivalent fuel value converted from the electric energy of the lithium ion battery and the supercapacitor unit; $C O(X), H C(X)$, and $N O_{x}(X)$ are the emission values of $\mathrm{CO}, \mathrm{HC}$, and $\mathrm{NO}_{x}$, respectively.

For the analysis of the fuel consumption of HEVs, in addition to the calculation of the fuel consumption of the internal combustion engine, the electric energy consumed by the lithium ion battery and the supercapacitor unit should also be converted to its equivalent fuel value $F C R$. The calculation equation is 


$$
F C R=\frac{F_{(b+u)}-F_{r b}}{l} \times 10^{-2},
$$

where $F C R$ is the equivalent fuel value converted from the electric energy of the lithium ion battery and the supercapacitor unit; $F_{(b+u)}$ is the equivalent fuel value of the total energy consumed by the lithium ion battery and the supercapacitor unit; $F_{r b}$ is the equivalent fuel value of the total energy recovered by the lithium ion battery and the supercapacitor unit during regenerative braking; $l$ is the driving distance of the HEV.

Fuel consumption and emission values interact with each other. The best emission index is likely to lead to reduced fuel economy. In the multiobjective optimization, to ensure the best fuel economy and emission performance of HEVs, the specific form of $J(X)$ is

$$
\begin{aligned}
J(X)= & \frac{\omega_{1}}{\text { Fuel }_{\text {eng }}{ }^{*}} \int \text { Fuel }_{\text {eng }}(t) d t+\frac{\omega_{2}}{F C R^{*}} \int F C R(t) d t \\
& +\frac{\omega_{3}}{C O^{*}} \int C O(t) d t+\frac{\omega_{4}}{H C^{*}} \int H C(t) d t+\frac{\omega_{5}}{N O_{x}^{*}} \int N O_{x}(t) d t,
\end{aligned}
$$

where $\omega_{i}$ is the weight of the fuel consumption and emission values of the HEV; Fuel ${ }_{\text {eng }}{ }^{*}$ and $F C R^{*}$ are customized fuel consumption targets; $C \mathrm{O}^{*}, \mathrm{HC}^{*}$, and $\mathrm{NO}_{x}{ }^{*}$ are target emission values. In this paper, the federal emission regulations ${ }^{(18)}$ are selected, and the target values and weights adopted are shown in Table 1.

\section{Experimental Parameter Setting}

The initial population is represented by a binary value, and the length of the coding string directly affects the optimization degree of the target value. In different cases, the selected encoding length $L$ is different. When the membership function parameters are encoded by symbols, the coding method of the problem is used to determine the length $L$; when membership function parameters are encoded by floating-point numbers, the number of decisions determines the length $L$. In this paper, $L=112$.

The size of the initial population $M$ represents the number of parameters in the population to be optimized. When the number of parameters to be optimized is small, the operation speed of the genetic algorithm is improved but the diversity of the population is reduced; when there are many parameters in the population to be optimized, the difficulty of calculating the

Table 1

Objective values and weights.

\begin{tabular}{lcc}
\hline & Objective value & Weight \\
\hline Fuel $_{\text {eng }}{ }^{*}$ & $7.5 \mathrm{~L} / 100 \mathrm{~km}$ & 0.5 \\
CCR $^{*}$ & $4.3 \mathrm{~L} / 100 \mathrm{~km}$ & 0.2 \\
CO $^{*}$ & $1 \mathrm{~g} / \mathrm{km}$ & 0.1 \\
$\mathrm{HC}^{*}$ & $0.1 \mathrm{~g} / \mathrm{km}$ & 0.1 \\
NO $_{x}{ }^{*}$ & $0.08 \mathrm{~g} / \mathrm{km}$ & 0.1 \\
\hline
\end{tabular}


optimization process of the parameters to be optimized is increased, reducing the operation efficiency of the genetic algorithm. The population size $M$ selected in this paper is 100 .

\subsection{Design of genetic operators}

Genetic operators include the selection operator, crossover operator, and mutation operator. For the selection operation, we adopt the roulette proportion selection strategy, ${ }^{(19)}$ and the probability $P\left(X_{i}\right)$ of the selected parameters to be optimized is

$$
P\left(X_{i}\right)=f\left(X_{i}\right) / \sum_{i=1}^{M} f\left(X_{i}\right),
$$

where $M$ is the size of the population; $f\left(X_{i}\right)$ is the fitness of the parameter to be optimized. Each feasible solution is proportional to its fitness. The larger the fitness value, the better the quality of the feasible solution.

After completing the design of the genetic operators, it is necessary to consider the setting of the number of termination iterations. When the genetic algorithm runs to the end of iteration $T$, it will stop running and select the optimal control parameters from the current population. Considering the complexity of the optimization process, we set the number of iterations before termination $T$ to 50 .

\subsection{Constraint conditions}

In order to optimize the fuel economy and emission of HEVs, the power performance index of HEVs and the constraints of lithium ion batteries and supercapacitor units must be qualified. The specific constraints are given in Table 2 .

If only the power performance constraints of the HEV are considered in the calculation of the objective function, then the energy of the lithium ion battery and the supercapacitor is zero at the end of the whole cycle. Therefore, the state of change (SOC) values and the power and energy of the lithium ion battery and the supercapacitor must be restricted. In practical operation, the safety ranges of the lithium ion battery and supercapacitor SOCs are generally $0.2-1$ and $0.5-1$, respectively.

In this paper, the difference between the lithium ion battery and supercapacitor SOCs at the beginning and end of the cycle is limited as follows.

Table 2

Constraints on power performance of HEVs.

\begin{tabular}{lc}
\hline Index name (unit) & Value \\
\hline $0-97 \mathrm{~km} / \mathrm{h}$ acceleration time $t_{1}(\mathrm{~s})$ & 13.7 \\
64-97 km/h acceleration time $t_{2}(\mathrm{~s})$ & 7.2 \\
Maximum gradient $(\%)$ & 27 \\
Maximum speed $(\mathrm{km} / \mathrm{h})$ & 0.1 \\
\hline
\end{tabular}




$$
\begin{gathered}
P_{\text {bat_min }} \leq P_{b a t} \leq P_{\text {bat_max }} \\
P_{u c_{-} \text {min }} \leq P_{u c} \leq P_{u c_{-} \text {max }} \\
E_{\text {bat_min }} \leq E_{\text {bat }} \leq E_{\text {bat_max }} \\
E_{u c_{-} \text {min }} \leq E_{u c} \leq E_{u c_{-} \text {max }}
\end{gathered}
$$

Here, $P_{b a t}$ and $P_{u c}$ are the powers of the lithium ion battery and supercapacitor, and $E_{b a t}$ and $E_{u c}$ are the energies of the lithium ion battery and supercapacitor, respectively.

\section{Experimental Results}

In order to verify the effectiveness of the HEV fuzzy control strategy optimized by the feature selection genetic algorithm combined with the HEV simulation model under the New European Driving Cycle (NEDC) and Urban Dynamometer Driving Schedule (UDDS) cycle conditions, the off-line simulation method is used to solve the optimal fuzzy controller parameters. The optimized fuzzy controller based on the feature selection genetic algorithm is applied to the fuzzy control strategy of the HEV, and the results are compared with those of the non-optimized HEV. The simulation results of the vehicle fuzzy control strategy are also compared.

\subsection{Comparison of SOC between lithium ion battery and supercapacitor before and after optimization}

Under the NEDC cycle condition, the changes in the SOC before and after optimization are shown in Fig. 2. In the figure, $S O C_{-}$bat 1 and $S O C_{-} u c 1$ are the pre-optimization curves and

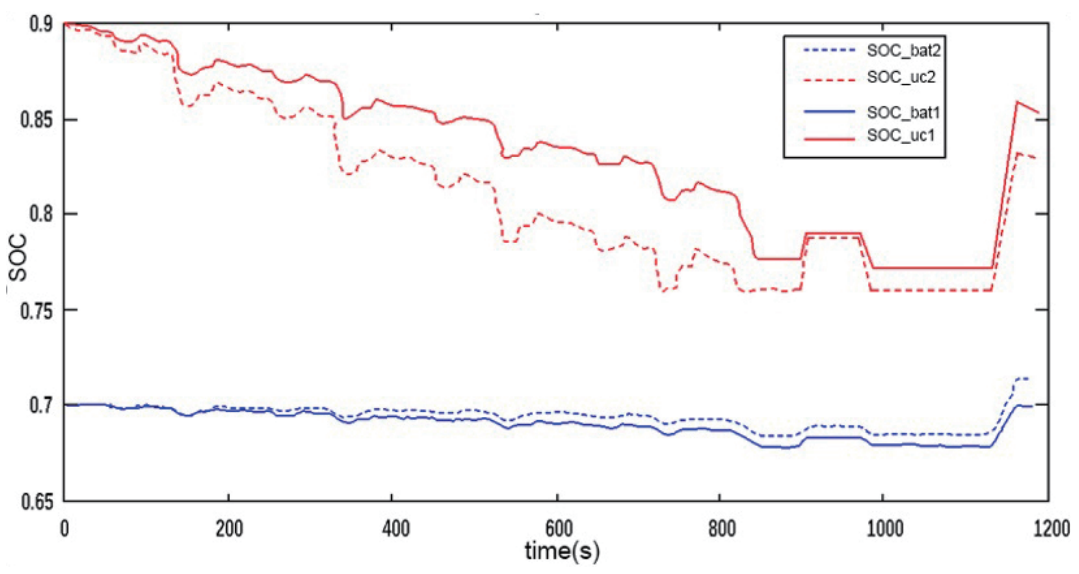

Fig. 2. (Color online) Comparison of SOC changes under NEDC driving cycle condition before and after optimization. 
SOC_bat2 and SOC_uc2 are the post-optimization curves. After optimization, the SOC_bat fluctuation of the lithium ion battery is small, the fluctuation of $S O C_{-} u c$ of the supercapacitor increases, and the effect of peak cutting and valley filling of the supercapacitor is strengthened.

\subsection{Fuel economy comparison of HEV before and after optimization}

Figures 3 and 4 respectively show the optimized fuel consumption curve of the HEV before and after optimization under the NEDC cycle condition.

The unit of the ordinates in Figs. 3 and 4 is g/s. Integrating the curves gives the total fuel consumption of the whole cycle, and integrating the NEDC gives the cycle mileage. We divided the total fuel consumption obtained by the above integration by the total driving mileage, then converted it into the fuel consumption per $100 \mathrm{~km}$. According to Figs. 3 and 4, under the NEDC cycle condition, the total fuel consumption of the vehicle is $6.253 \mathrm{~L} / 100 \mathrm{~km}$ under the fuzzy control strategy optimized by the feature selection genetic algorithm. Compared with the fuzzy control strategy before optimization $(6.856 \mathrm{~L} / 100 \mathrm{~km})$, the fuel consumption is reduced by $8.8 \%$.

Figures 5 and 6 respectively show the optimized fuel consumption curve of the HEV before and after optimization under the UDDS cycle condition.

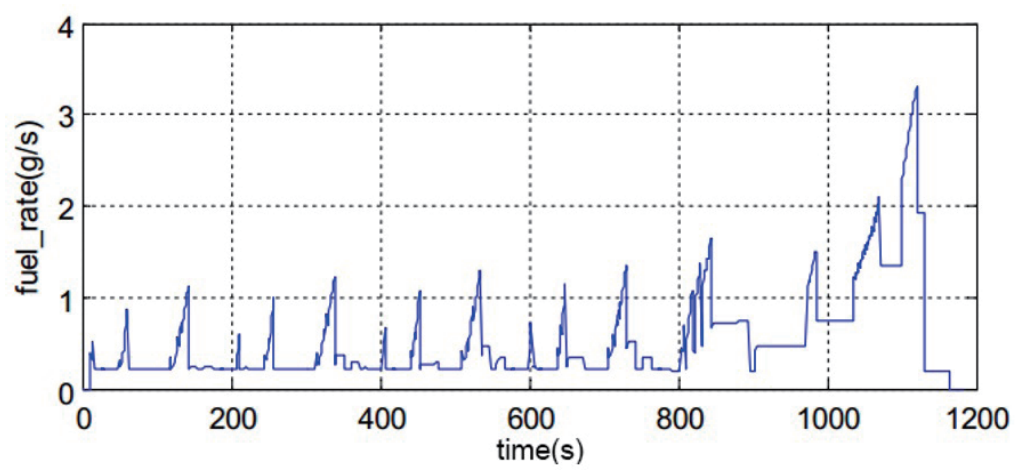

Fig. 3. (Color online) Fuel consumption curve before optimization under NEDC driving cycle condition.

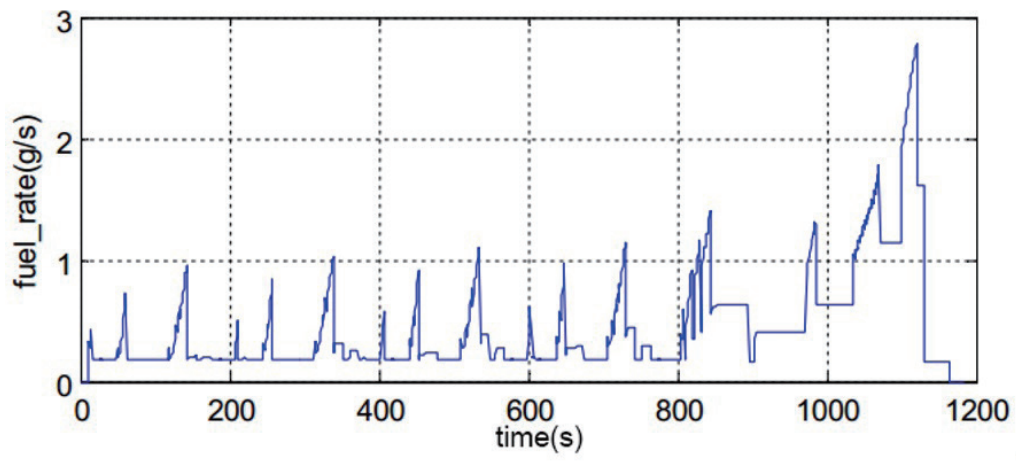

Fig. 4. (Color online) Fuel consumption curve after optimization under NEDC driving cycle condition. 


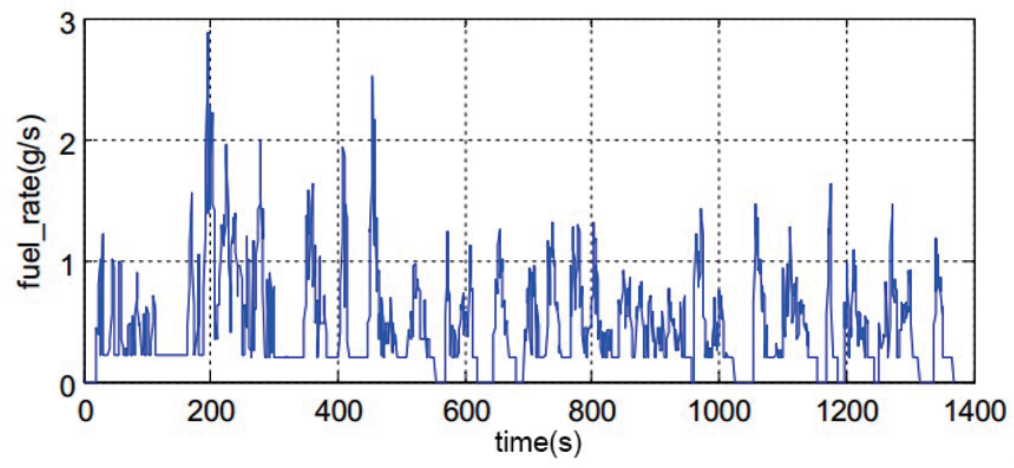

Fig. 5. (Color online) Fuel consumption curve before optimization under UDDS driving cycle condition.

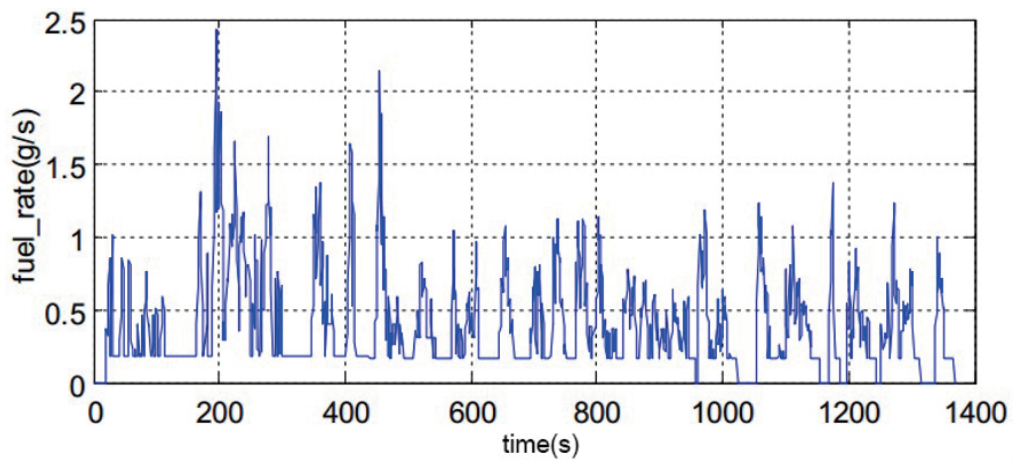

Fig. 6. (Color online) Fuel consumption curve after optimization under UDDS driving cycle condition.

From Figs. 5 and 6, it can be concluded that under the UDDS cycle condition, the fuel consumption of the whole vehicle is $6.624 \mathrm{~L} / 100 \mathrm{~km}$ under the fuzzy control strategy optimized by the feature selection genetic algorithm. Compared with the fuzzy control strategy before optimization $(7.320 \mathrm{~L} / 100 \mathrm{~km})$, the fuel consumption is reduced by $9.5 \%$. The results show that the fuel economy of the HEV has been greatly improved compared with the original fuzzy control strategy, and the effectiveness of the improved fuzzy logic control strategy based on the feature selection genetic algorithm is verified.

\subsection{Emission comparison of HEVs before and after optimization}

Figures 7 and 8 respectively show the emission curve of the HEV before and after optimization under the NEDC cycle condition. Figures 9 and 10 respectively show the emission curve of the HEV before and after optimization under the UDDS cycle condition. From Figs. 7-10, the optimized emission curve of the HEV under the NEDC and UDDS cycle conditions was obtained. The specific emission value data is shown in Table 3.

It can be seen from Table 3 that under the NEDC cycle condition, the emission values of $\mathrm{HC}$, $\mathrm{CO}$, and $\mathrm{NO}_{x}$ of the HEV optimized by the feature-based genetic algorithm are $0.244,1.117$, and 


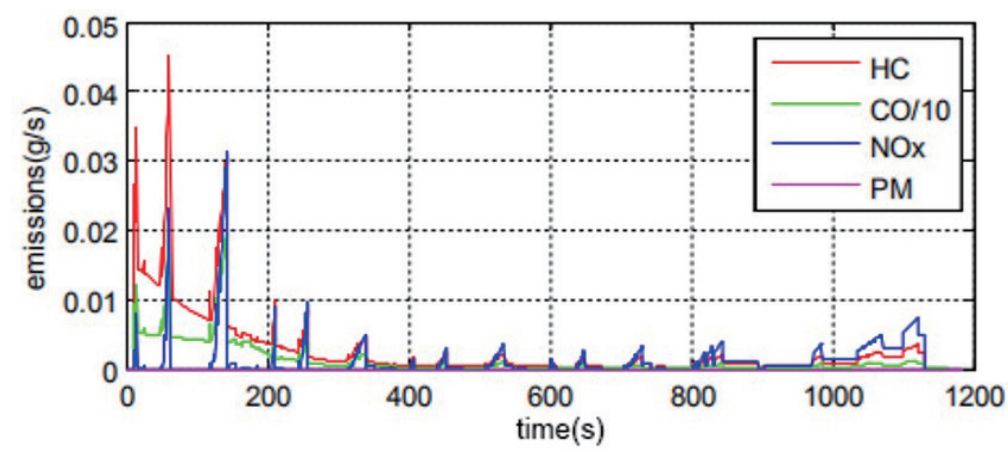

Fig. 7. (Color online) Emission curve before optimization under NEDC driving cycle condition.

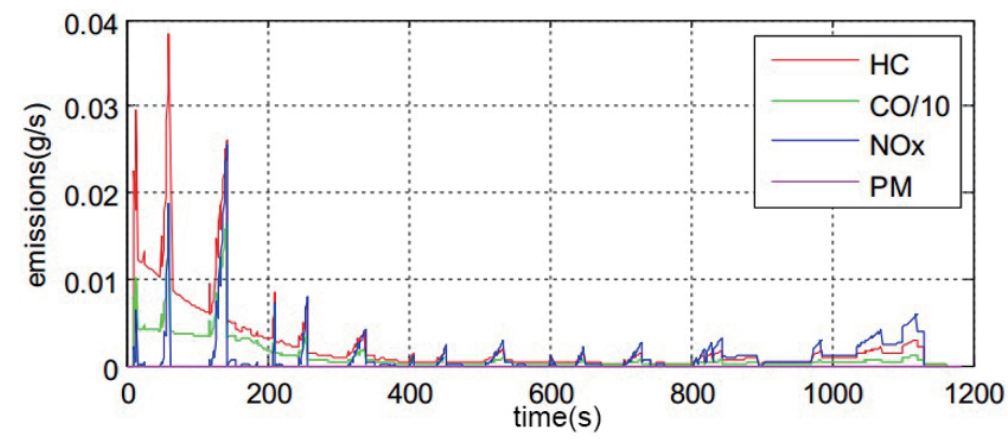

Fig. 8. (Color online) Emission curve after optimization under NEDC driving cycle condition.

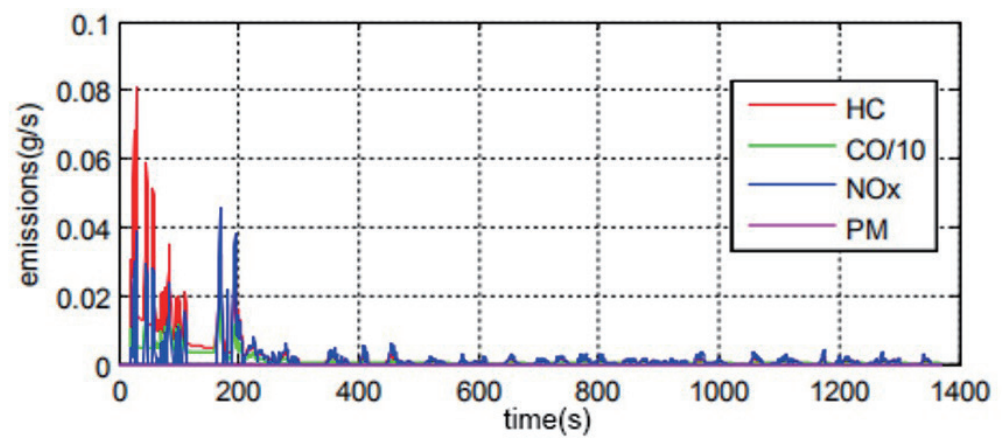

Fig. 9. (Color online) Emission curve before optimization under UDDS driving cycle condition.

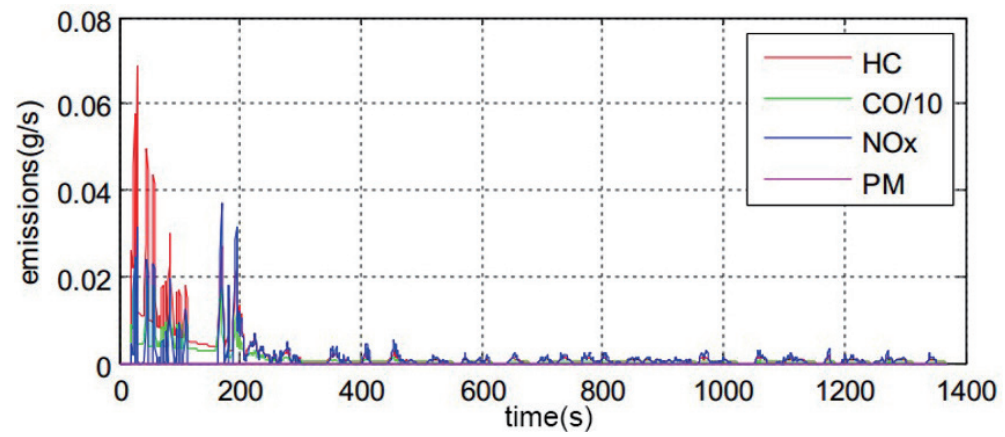

Fig. 10. (Color online) Emission curve after optimization under UDDS driving cycle condition. 
Table 3

Emission values after fuzzy control optimization.

\begin{tabular}{lcccccc}
\hline Driving cycle & \multicolumn{3}{c}{ Emission values $(\mathrm{g} / \mathrm{km})$} & \multicolumn{3}{c}{ Optimal emission values $(\mathrm{g} / \mathrm{km})$} \\
\hline \multirow{2}{*}{ NEDC } & $\mathrm{HC}$ & $\mathrm{CO}$ & $\mathrm{NO}_{x}$ & $\mathrm{HC}$ & $\mathrm{CO}$ & $\mathrm{NO}_{x}$ \\
\cline { 2 - 7 } & 0.286 & 1.329 & 0.123 & 0.244 & 1.117 & 0.101 \\
\hline \multirow{2}{*}{ UDDS } & $\mathrm{HC}$ & $\mathrm{CO}$ & $\mathrm{NO}_{x}$ & $\mathrm{HC}$ & $\mathrm{CO}$ & $\mathrm{NO}_{x}$ \\
\cline { 2 - 7 } & 0.295 & 1.524 & 0.148 & 0.256 & 1.317 & 0.125 \\
\hline
\end{tabular}

$0.101 \mathrm{~g}$, respectively. Compared with the optimized vehicle fuzzy control strategy, the emission values of $\mathrm{HC}, \mathrm{CO}$, and $\mathrm{NO}_{x}$ are reduced by $14.7,15.9$, and $17.9 \%$, respectively. Under the UDDS cycle condition, the emission values of $\mathrm{HC}, \mathrm{CO}$, and $\mathrm{NO}_{x}$ of the $\mathrm{HEV}$ optimized by the feature selection genetic algorithm are $0.256,1.317$, and $0.125 \mathrm{~g}$, respectively. Compared with the vehicle fuzzy control strategy before optimization, the emission values of $\mathrm{HC}, \mathrm{CO}$, and $\mathrm{NO}_{x}$ are reduced by $13.2,13.6$, and $15.5 \%$, respectively.

\section{Conclusions}

We proposed a novel fuzzy control strategy of HEVs based on the feature selection genetic algorithm, which greatly shortens the selection time of the optimization parameters of the traditional genetic algorithm.

Firstly, feature selection based on the genetic algorithm of multivariate data was proposed. The proposed algorithm first evaluates the features of the dimension, adjusts the weight of each feature according to the difference between similar nearest neighbors and dissimilar nearest neighbors of multivariate data, and guides the search of the genetic algorithm based on the feature weight so as to improve the search ability of the proposed algorithm and the accuracy of the obtained features. Then, the fitness (or objective) function of the features is calculated by combining the feature weights, and the fitness is used as the evaluation index to start the genetic algorithm.

Finally, the performances of the fuzzy control strategy of the HEV and the fuzzy control strategy optimized by the feature selection genetic algorithm under the NEDC and UDDS cycle conditions were analyzed and compared. The results show that the novel fuzzy control greatly improves the fuel economy of the HEV, reducing the emission values, causes the SOC of the lithium ion battery to fluctuate in a small range, and causes the SOC of the supercapacitor to fluctuate in a large range. The improved fuzzy control plays an enhanced role in the peak cutting and valley filling of the supercapacitor, ensures that the lithium ion battery basically works in a constant-current output mode, and optimizes the lithium separation. The discharging process of the sub-battery verifies the effectiveness of the optimized fuzzy control strategy and realizes the control function of the vehicle controller.

\section{Acknowledgments}

This work was supported in part by the Teaching Quality and Reform of Higher Vocational Education Project of Guangdong Province (GDJG2019463), Hebei Provincial High Level Talents 
Foundation of Hebei Province (A2016002025), Hebei Province Key R\&D Project (17394501D), Natural Science Foundation of Characteristic Innovation Project of Guangdong Ordinary University and the Science and Technology Research Project of Hebei Higher Education (ZD2017213), subsidized projects for Overseas Students (CL201705), Zhaoqing Research and Development Technology and Application of Energy Conservation and Environmental Protection Ecological Governance (2020SN004), and Characteristic Innovation Projects of Colleges and Universities in Guangdong Province (2019KTSCX201).

\section{References}

1 A. Panwar, G. Sharma, and R C. Bansal: Electr. Power Compon. Syst. 4 (2019) 1. https://doi.org/10.1080/15325 $\underline{008.2019 .1659452}$

2 T. Zhu, H. Zheng, and Z. Ma: Adv. Mech. Eng. 11 (2019) 1. https://doi.org/10.1177/1687814019833500

3 C. Yin, S. Wang, and C. Yu: Adv. Mech. Eng. 11 (2019) 56. https://doi.org/10.1177/1687814019830797

4 M. Pang, Y. Shi, and W. Wang: Energy Explor. Exploit. 37 (2019) 558. https://doi.org/10.1177/0144598718784036

5 M. Ghanamijaber: Evolving Syst. 10 (2019) 273. https://doi.org/10.1007/s12530-018-9228-X

6 Q. Xu, X. Luo, and X. Jiang: IET Electr. Syst. Transp. 12 (2018) 502. https://doi.org/10.1049/iet-est.2017.0067

7 X. Yuan, J. Peng, and Z. Guopeng: Appl. Rese. Comp. 12 (2019) 345. https://doi.org/10.19734/ j.issn.1001-3695.2018.06.0395

8 F. Lei, Y. Bai, and W. Zhu: J. Energy 167 (2019) 1040. https://doi.org/10.1016/j.energy.2018.11.052

9 G. I. Y. Mustafa, H. P. Wang, and Y. Tian: Adv. Eng. Software 127 (2019) 141. https://doi.org/10.1016/ j.advengsoft.2018.04.009

10 D. Lal and A. Barisal: Recent Adv. Electr. Electr. Eng. 12 (2019) 519. https://doi.org/10.2174/235209651166618 0717142058

11 F. Yi, H. Hu, and D. Zhou: Electri. Mach. Cont. 7 (2003) 54. https://doi.org/10.1016/12172117.2018.11.052

12 I. Lucio, L. Vincenzo, and S. Pierluigi: Fuzzy Opt. Deci. Maki. 2 (2003) 359. https://doi. org $/ 10.1016 / 1000034827132410$

13 J. I. Guerrero, E. Personal, and G. Antonio: Energies 12 (2019) 2402. https://doi.org/10.3390/en12122402

14 W. Gao and C. Mi: Int. J. Electr. Hybrid Veh. 1 (2007) 57. https://doi.org/10.1504/IJEHV.2007.014447

15 Y. Tian, X. Zhang, and L. Zhang: Proc. 2009 2nd Int. Conf. Intelligent Computation Technology and Automation (IEEE, 2009) 720-723. https://doi.org/10.1109/ICICTA.2009.409

16 Y. Li: J. Changsha Univ. 30 (2016) 58. https://doi.org/10.1016/ 201701008

17 Q. Zeng and J. Huang: Proc. 2007 IEEE Int. Conf. Automation and Logistics (IEEE, 2007) 908-912. https:// doi.org/10.1109/ISDA.2006.252

18 W. Li, Y. Yin, and R. Zhang: Standard Sci. 9 (2017) 28. https://doi.org/10.2172/814411 